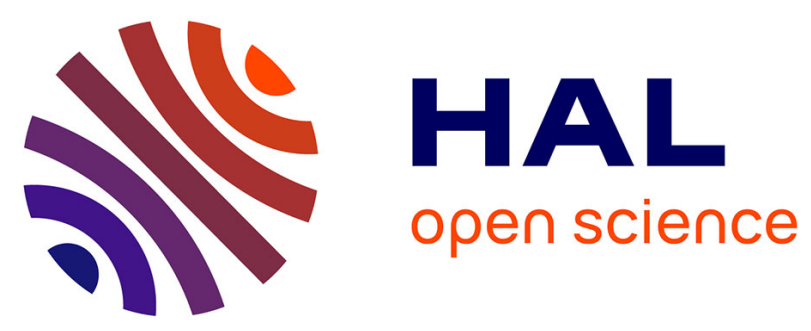

\title{
Caractérisation et prévision des sécheresses et étiages en France à partir de la chaîne hydrométéorologique Safran-Isba-Modcou
}

Jean-Michel Soubeyroux, Jean-Philippe Vidal, M. Baillon, M. Blanchard, J.P. Céron, L. Franchistéguy, F. Régimbeau, E. Martin, J.C. Vincendon

\section{To cite this version:}

Jean-Michel Soubeyroux, Jean-Philippe Vidal, M. Baillon, M. Blanchard, J.P. Céron, et al.. Caractérisation et prévision des sécheresses et étiages en France à partir de la chaîne hydrométéorologique Safran-Isba-Modcou. La Houille Blanche - Revue internationale de l'eau, 2010, 5, p. 30 - p. 39. 10.1051/lhb/2010051 . hal-00593584

\section{HAL Id: hal-00593584 \\ https://hal.science/hal-00593584}

Submitted on 16 May 2011

HAL is a multi-disciplinary open access archive for the deposit and dissemination of scientific research documents, whether they are published or not. The documents may come from teaching and research institutions in France or abroad, or from public or private research centers.
L'archive ouverte pluridisciplinaire HAL, est destinée au dépôt et à la diffusion de documents scientifiques de niveau recherche, publiés ou non, émanant des établissements d'enseignement et de recherche français ou étrangers, des laboratoires publics ou privés. 


\title{
Caractérisation et prévision des sécheresses et étiages en France à partir de la chaîne hydrométéorologique Safran-Isba-Modcou
}

\author{
Characterizing and forecasting droughts and low-flows in France with \\ the Safran-Isba-Modcou hydrometeorological suite
}

JEAN-MICHEL SOUBEYROUX

Météo-France, Direction de la Climatologie

42 avenue Coriolis 31057 Toulouse Cedex 1, France

Tél : +33 (0) 5610783 03, Fax : +33 (0)5 610783 09, e-mail : jean-michel.soubeyroux@meteo.fr

\author{
JEAN-PHILIPPE VIDAL \\ Cemagref, HHLY \\ Unité de Recherche Hydrologie-Hydraulique \\ 3 bis quai Chauveau, CP 220, 69336 Lyon Cedex 09 \\ Tél : +33 (0)4 722089 45, Fax : +33 (0)4 7847 78 75, e-mail : jean-philippe.vidal@cemagref.fr
}

\begin{abstract}
MARTINE BAILLON, MICHÈLE BLANCHARD, JEAN-PIERRE CÉRON, LAURENT FRANCHISTÉGUY, FABIENNE RÉGIMBEAU
\end{abstract}

Météo-France, Direction de la Climatologie

\section{ERIC MARTIN}

CNRM/GAME, Météo-France et CNRS

42 avenue Coriolis 31057 Toulouse Cedex 1, France

Tél : +33 (0) 5610785 62, Fax : +33 (0)5 61078309

\section{JEAN-CHRISTOPHE VINCENDON}

Météo-France, Direction de la Prévision

42 avenue Coriolis 31057 Toulouse Cedex 1, France

Tél : +33 (0) 5610782 03, Fax : +33 (0)5 61078209

$E$

nliaison avec les évènements de sècheresse en Métropole, les applications hydrométéorologiques ont bénéficiéces dernières années des actions de recherche sur la modélisation des bilans hydriques et notamment le développement de la chaîne Safran-Isba-Modcou (SIM) à Météo-France en lien avec ses partenaires hydrologiques.

La chaîne SIM est maintenant largement utilisée pour le suivi opérationnel de la ressource en eau en France. Elle a fait l'objet également d'une réanalyse à haute résolution spatiale sur la période août 1958 - juillet 2008 et a permis l'élaboration de différents indicateurs de sècheresses pour la caractérisation des événements passés de déficit hydrique sur la France dans les différentes composantes du cycle de l'eau. Ces indicateurs développés dans le cadre du projet ClimSec ont vocation à une large utilisation pour les applications opérationnelles. En matière de prévision numérique du temps, des approches spécifiques, basées sur la modélisation des téléconnexions planétaires océan-atmosphère, sont en cours de développement pour apporter des informations utiles au suivi de la ressource en eau aux différentes échéances étendues (semaine, mois, saison). Des espoirs importants se portent actuellement sur la prévision saisonnière hydrologique à travers les travaux de couplage de la chaîne SIM avec le système de prévision saisonnière Arpège Climat de Météo-France. L'ensemble de ces applications fait l'objet d'expérimentations régulières avec les services hydrologiques de l'État pour faciliter leur prise en compte progressive dans les instances de suivi des sècheresses.

$H$ ydrometeorological applications have recently benefited in France from research on water balance modelling, including the development of the Safran-Isba-Modcou suite (SIM) at Météo-France with hydrological partners. The SIM suite is now widely used for operational monitoring of water resources in France. A high spatial resolution reanalysis over the period August 1958-July 2008 has allowed the development of various 
drought indicators characterizing past events of water deficit in France within the different components of the water cycle. These indicators developed within the ClimSec project are intended to be used for operational applications. In terms of numerical weather prediction, specific approaches based on the understanding of global ocean-atmosphere teleconnections are being developed in order to provide useful information for water resource monitoring at different time ranges (week, month, season). High expectations currently focus on the seasonal hydrologic forecast through the work of coupling the SIM suite with the forecasting seasonal system Arpege Climat of Mété-France. All these applications are being tested with national hydrological services to facilitate their gradual use as decision support for drought committees.

\section{I $\square$ INTRODUCTION}

Les enjeux du suivi de la ressource en eau et notamment l'anticipation des situations de rareté, voire de pénurie, réclament de nos jours la mise en place d'applications hydrologiques opérationnelles spécifiques, au même titre que celles développées ces dernières années pour la prévision des crues. Ces applications doivent en premier lieu permettre de qualifier le caractère anormal de chaque composante du cycle de l'eau dont les déficits peuvent induire des conséquences particulières sur la disponibilité de la ressource. Les attentes opérationnelles concernent aussi la mise à disposition de scénarios prévisionnels de l'évolution de la situation à des horizons temporels éloignés (mensuel à saisonnier) adaptés aux différentes problématiques d'usages de la ressource.

À Météo-France, le développement de la modélisation spatialisée des bilans hydriques à partir de la chaîne Safran-IsbaModcou (SIM) sur l'ensemble de la France a permis d'enrichir le suivi hydrométéorologique traditionnel qui s'appuyait notamment sur la qualification des déficits pluviométriques calculés à partir des longues chroniques d'observations sur le territoire. Ainsi, l'information sur la teneur en eau des sols superficiels issue de la chaîne SIM est devenue depuis 2005 un élément majeur de qualification de l'état hydrique en Métropole [1], dans le cadre du Bulletin de Situation Hydrologique (BSH) édité par le MEEDDM. Mais le besoin de caractérisation de la situation hydrologique et notamment des sècheresses nécessite aussi la mise en place d'indicateurs spécifiques. Ce constat, renforcé par les récents travaux sur le changement climatique soulignant l'augmentation du risque de sècheresse en Métropole, a motivé la mise en place du projet ClimSec financé par la Fondation MAIF et MétéoFrance $[2,3]$, consacré à la caractérisation des sècheresses sur la période 1958-2008 à partir de la chaîne SIM. Enfin, les progrès de modélisation du système climatique planétaire et notamment des interactions océan-atmosphère permettent d'envisager des applications opérationnelles de prévision aux échéances mensuelles et saisonnières et des couplages hydrométéorologiques visant à combiner les différentes sources de prévisibilité des composantes du système hydroclimatique.

Cet article présentera les apports de la modélisation hydrologique SIM pour la caractérisation des sècheresses et étiages en France, notamment à travers les résultats opérationnels attendus du projet ClimSec et les progrès récents réalisés en matière de prévision météorologique aux échéances étendues débouchant sur des perspectives nouvelles en matière de prévision saisonnière hydrologique

\section{II — LE SUIVI HYDROMÉTÉOROLOGIQUE OPÉRATIONNEL AVEC LE MODÈLE SIM}

\section{II.1 PRÉSENTATION DU MODÈLE HYDROMÉTÉOROLOGIQUE SIM}

Cette application hydrométéorologique est constituée de l'enchaînement de 3 modèles, dont l'extension à l'ensemble de la France a fait l'objet de nombreuses validations [4] :

- Les variables atmosphériques (précipitations liquide et neigeuse, température, rayonnements solaire et infrarouge, vent et humidité de l'air) sont analysées par le système d'analyse Safran [5], qui prend en compte l'ensemble des observations disponibles, des analyses du modèle opérationnel Arpège, et des données climatiques, y compris le relief, pour restituer des champs au pas de temps horaire à une résolution de $8 \times 8 \mathrm{~km}^{2}$ sur l'ensemble du territoire français ;

- Le modèle Isba (Interactions-Sol-Biosphère-Atmosphère), schéma de surface utilisé ici dans sa version hydrologique à 3 couches, calcule les bilans d'eau et d'énergie sur l'ensemble sol-végétation, en prenant en compte la composante nivale (très importante sur les bassins montagneux), au pas de temps de 5 minutes. Isba prend en compte de manière explicite les paramètres de texture de sol (base des sols de l'INRA à $1 \mathrm{~km}$ ) et le type de végétation (base Ecoclimap, modifiée sur la France pour distinguer les types de cultures ayant différents cycles annuels) ;

- Enfin, le modèle Modcou, développé par l'École des Mines de Paris, simule le niveau des nappes, leurs interactions avec les rivières, et les débits quotidiens sur plus de 900 stations en France.

\section{II.2 FONCTIONNEMENT OPÉRATIONNEL POUR LE SUIVI HYDROMÉTÉOROLOGIQUE NATIONAL}

La chaîne SIM qui fonctionne dans l'environnement opérationnel de Météo-France depuis décembre 2003 est activée selon deux modes :

- une première analyse est réalisée en temps " quasi » réel (traitement quotidien chaque jour à $9 \mathrm{~h}$ de la période $\mathrm{J}-1,6 \mathrm{~h}$ à $\mathrm{J}, 6 \mathrm{~h}$ ) en utilisant les observations disponibles. L'analyse quotidienne « temps réel » de précipitations utilise environ 1500 postes de mesures pluviométriques par jour sur le territoire ; 

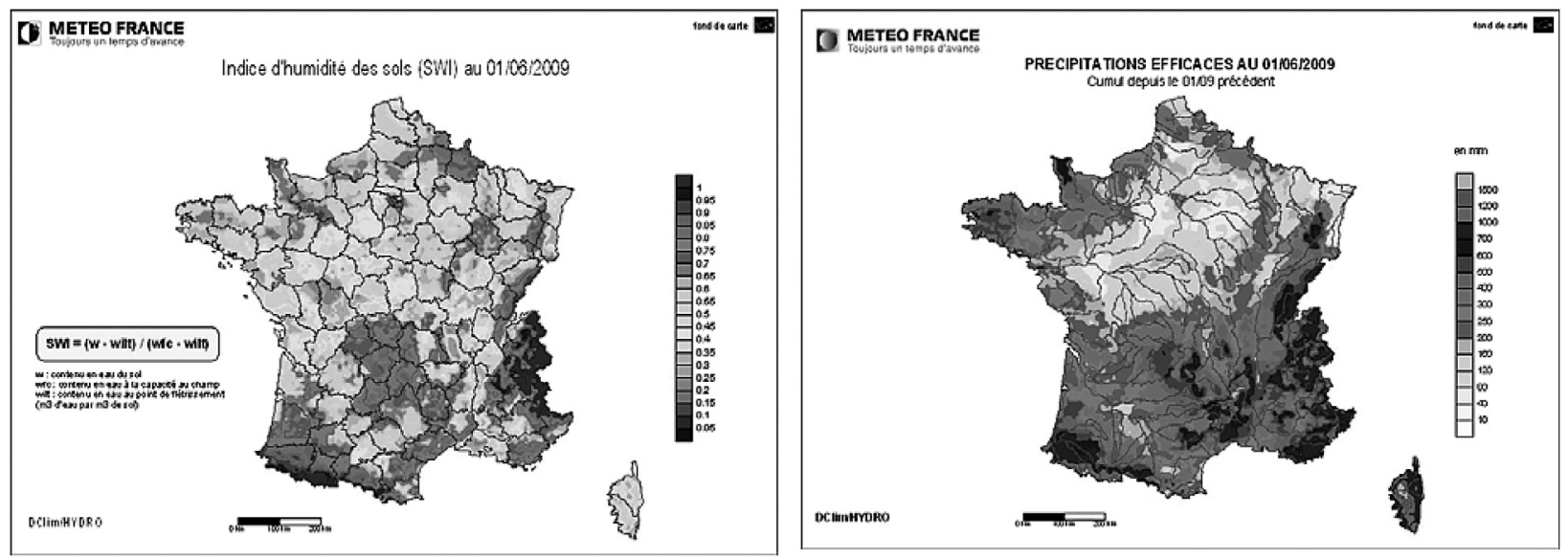

Figure 1 : Carte d'indice d'humidité des sols (SWI) sur la France au $1^{\text {er }}$ juin 2009 (à gauche) et de pluies efficaces au $1^{\text {er }}$ mai 2009 cumulées depuis le $1^{\text {er }}$ septembre précédent (à droite).

- une deuxième analyse est réalisée ensuite en temps différé $(\mathrm{J}+45)$, afin de bénéficier de l'ensemble des données du réseau climatologique d'État, soit environ 4000 postes pluviométriques.

Cette modélisation permet de constituer une base de données des paramètres météorologiques interpolés par Safran mais aussi des termes du bilan hydrique issus d'Isba, constituant ainsi une analyse optimale présente et passée du bilan hydrique sur la France, bénéficiant de l'ensemble des observations climatologiques au sol et des différentes ébauches issus des modèles atmosphériques. Une troisième analyse a été menée récemment, tirant partie de la réanalyse ERA40 réalisée par le Centre Européen pour les Prévisions Météorologiques à Moyen Terme (CEPMMT) pour les variables atmosphériques depuis 1958. La réanalyse du modèle Safran a ainsi pu être mise en œuvre et validée sur la période allant d'août 1958 à nos jours [6] et étendue aux modèles Isba et Modcou.

De nombreuses applications ont été mises en place en matière d'hydroclimatologie opérationnelle ou de recherche [7], ainsi qu'en prévision des crues [8]. Ces différentes analyses trouvent logiquement une valorisation particulièrement pertinente pour le suivi de la ressource en eau. Ainsi, pour la contribution météorologique au BSH national, produit par le MEEDDM, Météo-France fournit depuis 2005, les cartes et commentaires pour les rubriques « eau dans le sol» et « manteau neigeux » sur les domaines Alpes et Pyrénées, pour les mois d'avril et mai.

L'indice d'humidité des sols, le Soil Wetness Index (SWI), est estimé et cartographié quotidiennement ainsi que son écart par rapport à la climatologie du modèle SIM. La combinaison des 2 cartes est très utile lors des comités sècheresse. La figure 1 (gauche) montre qu'au $1^{\text {er }}$ juin 2009 les sols étaient généralement bien humides sur la France à l'exception de quelques zones : Poitou-Charentes, Bourgogne et Vallée du Rhône. La contribution météorologique au BSH national s'est enrichie en 2008 avec l'alimentation de la rubrique « pluies efficaces ». Les pluies efficaces, calculées à partir des précipitations totales (pluie et neige) issues de
Safran diminuées de l'évapotranspiration réelle issue d'Isba, permettent d'évaluer la contribution des pluies à la recharge des nappes. La figure 1 (droite) montre un cumul de pluie efficace plus important lors de la période de recharge 20082009 sur la moitié sud de la France que sur la moitié nord (hors Bretagne).

Pour les Alpes et les Pyrénées, des cartes de l'équivalent en eau du manteau neigeux et de son écart à la normale complètent l'analyse en permettant d'évaluer la quantité d'eau encore disponible sous forme de neige à l'approche de la période d'étiage. La figure 2 présente un exemple sur les Pyrénées au printemps 2009.

\section{III — CARACTÉRISATION DES SÉCHERESSES PASSÉES}

\section{- III.1 DÉFINITION DES INDICES DE SÈCHERESSE}

Dans le cadre du projet ClimSec, Safran a tout d'abord été mis en œuvre et validé sur la période 1958-2008, puis cette réanalyse atmosphérique a servi de point d'entrée à la simulation hydrologique pour générer en particulier des valeurs de SWI à la résolution de $8 \mathrm{~km}$ sur la France et de débit au droit de 900 stations hydrométriques et de plus de 150 exutoires. La réanalyse de la chaîne SIM permet une approche très complète de la notion de sécheresse, définie comme un déficit en eau d'une composante (au moins) du cycle hydrologique [9]. En effet, la chaîne SIM permet de bénéficier d'un calcul des variables cohérent tout au long du cycle hydrologique pour appréhender les différents types de sécheresses - météorologiques, agricoles et hydrologiques liées aux différentes composantes du cycle.

L'approche adoptée pour la définition d'indices de sécheresse s'inspire du Standardized Precipitation Index (SPI), largement utilisé au niveau international dans les études mais aussi en mode opérationnel, par exemple aux Etats-Unis, Le SPI a par ailleurs été reconnu comme indice universel pour 
Domaine Pyrénées

Analyse ISBA du 01/05/2009

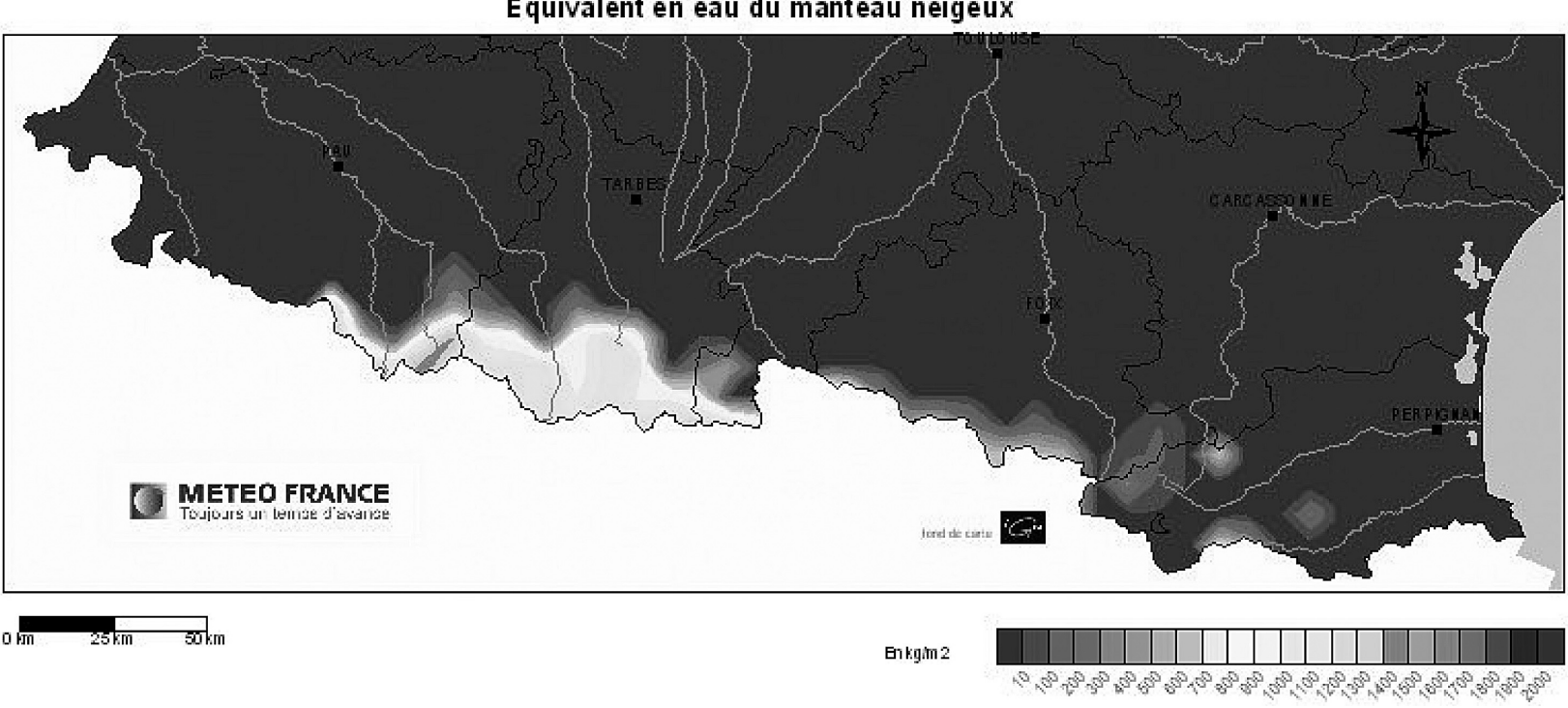

Figure 2 : Carte d'équivalent en eau du manteau neigeux sur les Pyrénées au $1^{\mathrm{er}}$ mai 2009.

l'analyse des sècheresses météorologiques par l'OMM en décembre 2009 [10]. La variable considérée au pas de temps mensuel - précipitations totales, SWI ou débit -est cumulée/ moyennée sur $n$ mois glissants, puis les distributions statistiques de chaque mois calendaire sont ajustées sur des distributions continues. Le choix a été fait ici d'utiliser des estimateurs non paramétriques de densité à noyaux gaussiens pour tenir compte du caractère bimodal de certaines distributions de l'humidité du sol. Les distributions sont ensuite projetées sur une loi normale centrée réduite pour calculer les valeurs d'indices associées. Cette méthode assure donc une cohérence spatiale de l'indice, une équivalence valeur/probabilité de non-dépassement en référence à la climatologie locale, ainsi qu'une vision à différentes échelles de temps.

Trois types d'indices ont ainsi été calculés : le Standardized Precipitation Index lui-même, le Standardized Soil Wetness Index (SSWI) et le Standardized Flow Index (SFI) [11]. Le Tableau 1 présente la correspondance entre variables SIM, types de sécheresse et indices associés, et donne le domaine de calcul de ces différents indices sur la France. Chacun de ces indices a été calculé avec des échelles de temps variant de 1 à 24 mois. Dans la suite de l'article seront présentés uniquement des résultats pour l'échelle de temps 3 mois, donc à l'aide des indices SPI3, SSWI3 et SFI3.

\section{III.2 CARACTÉRISATION DES PÉRIODES SÈCHES}

L'évolution de chaque indice de sécheresse peut tout d'abord être examinée de manière globale sur la France en considérant la proportion du territoire affectée par la sécheresse, i.e. où l'indice est en-dessous d'un seuil donné. La Figure 3 présente l'évolution de cette surface pour les indices SPI3, SSWI3 et SFI3.

On peut ainsi reconnaître les périodes où la sécheresse affectait une part conséquente du territoire, par exemple l'année 1976 ou encore les trois années 1988 à 1990 . La Figure 3 met en avant la forte corrélation entre les déficits de chacune des composantes du cycle hydrologique, mais aussi des différences notables. On peut ainsi remarquer le déficit d'humidité du sol important et étendu durant l'année 2003 en raison des fortes valeurs d'évapotranspiration liées aux températures élevées.

La représentation spatiale mois par mois des valeurs des indices permet quant à elle de saisir l'évolution temporelle des surfaces affectées à différents degrés par la sécheresse. La Figure 4 présente l'évolution conjointe des trois indices de sécheresses à l'échelle de temps 3 mois entre mai et octobre 1976, période très sèche et nettement discernable sur la Figure 3. Les régions pour lesquelles les valeurs des

Tableau 1 : Correspondance entre variables SIM, types de sécheresses et indices associés et domaine de calcul.

\begin{tabular}{|l|l|l|l|l|}
\hline & \multicolumn{1}{|c|}{ Variable } & \multicolumn{1}{c|}{ Type de sécheresse } & \multicolumn{1}{c|}{ Indice } & \multicolumn{1}{c|}{ Domaine de calcul } \\
\hline Safran & Précipitations & Météorologique & SPI & 8602 mailles \\
\hline Isba & Humidité du sol & Agricole & SSWI & 8602 mailles \\
\hline Modcou & Débits & Hydrologique & SFI & 1163 stations/exutoires \\
\hline
\end{tabular}




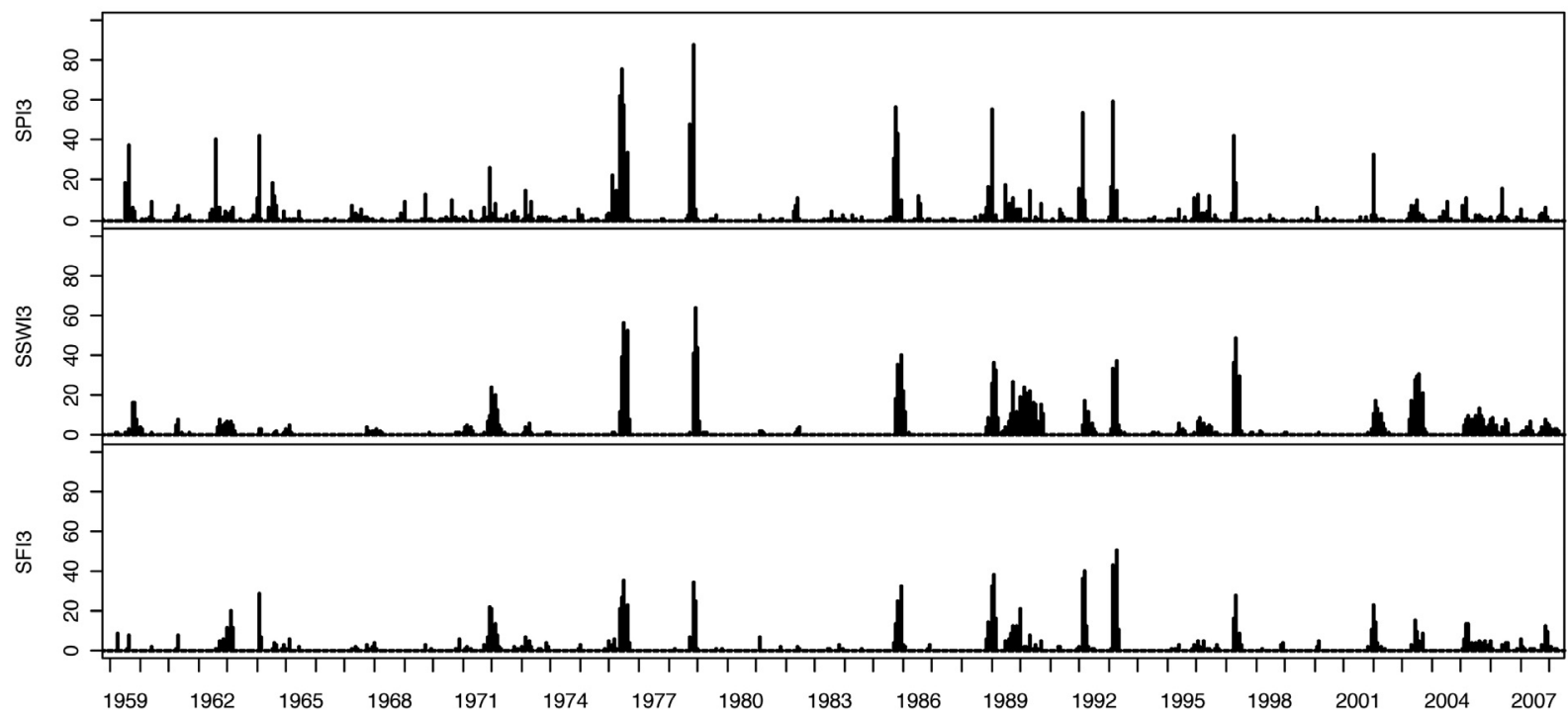

Figure 3 : Superficie affectée par la sécheresse, en \% de la France (SPI et SSWI) ou \% des stations (SFI). L'échelle de temps des indices est de 3 mois et le seuil utilisé pour l'état de sécheresse correspond à une probabilité de $5 \%$.
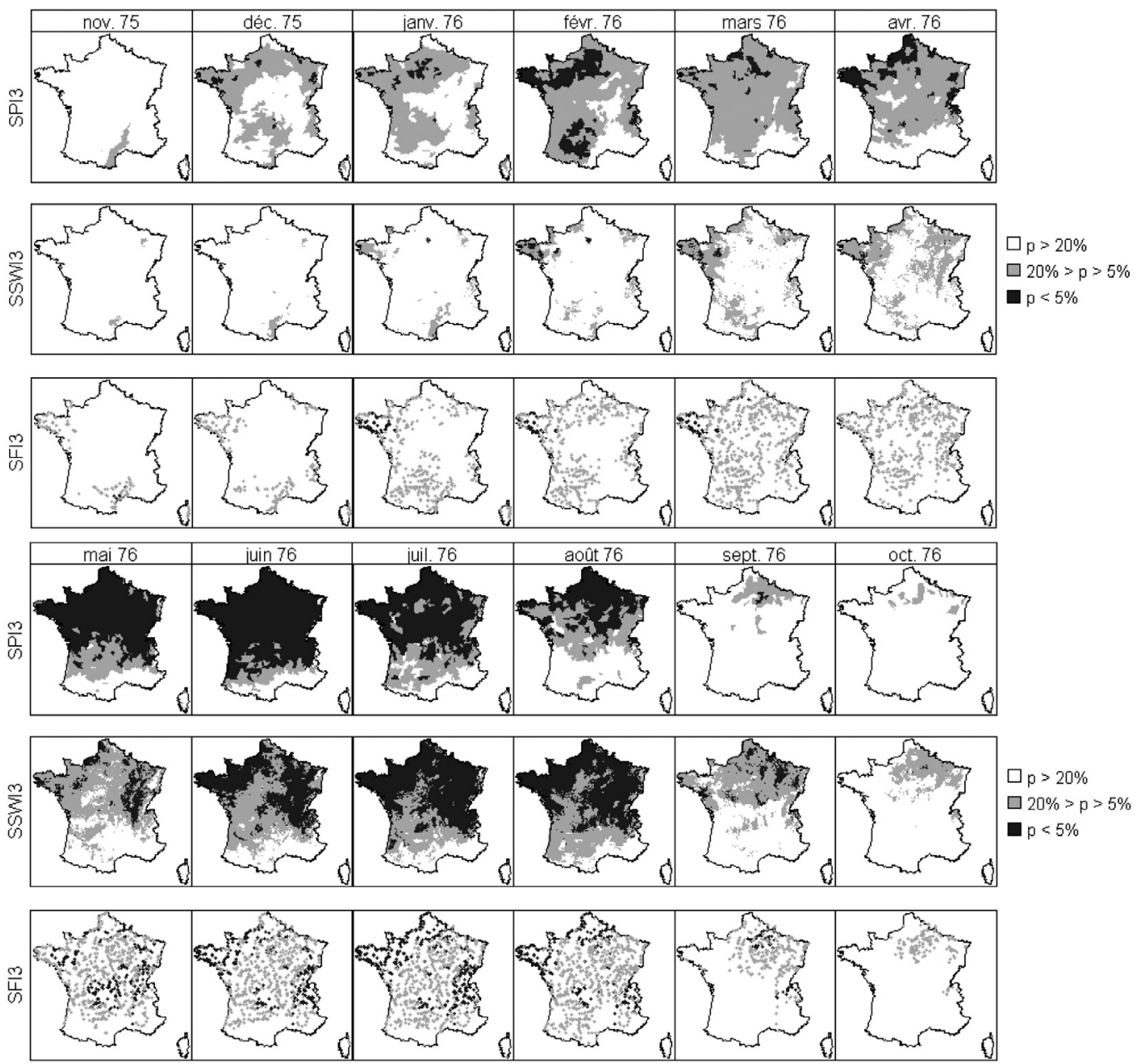

Figure 4 : Évolution de la sécheresse de 1976 avec les indices SPI3, SSWI3 et SFI3. La couleur gris foncé (resp. clair) représente les valeurs de probabilité d'occurence inférieure à $5 \%$ (resp. $20 \%$ ). Les valeurs de l'indice sont datées de la fin de l'échelle de temps considérée : les valeurs du SPI3 de mai 1976 correspondent ainsi aux déficits de précipitations sur la période mars 1976 - mai 1976. 


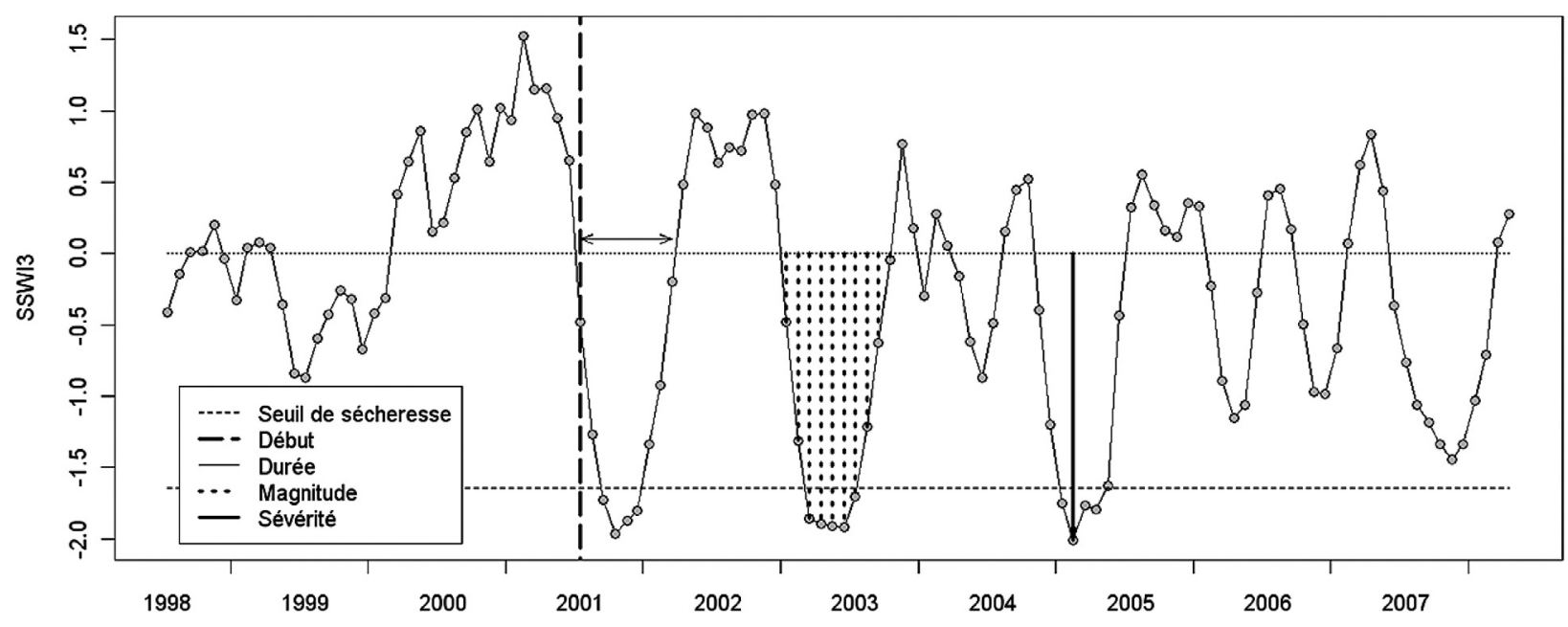

Figure 5 : Définition des caractéristiques locales des événements de sécheresse : début, durée, magnitude et sévérité. Exemple de l'évolution du SSWI3 sur 10 ans sur une maille située près de Toulouse avec un seuil fixé à $5 \%$.

indices étaient inférieures aux seuils d'occurrence de $20 \%$ et $5 \%$ sont représentées sur la Figure 4, qui montre tout d'abord que le pourtour méditerranéen a été largement épargné durant cet épisode.

On peut par ailleurs voir sur l'évolution du SPI3 que la sécheresse météorologique qui avait débuté en décembre 1975, a atteint un pic en juin 1976 pour se résorber rapidement en septembre. La sécheresse agricole est encore moins marquée que la sècheresse météorologique en mai 1976, montrant par là la résistance du sol et de la végétation aux déficits de précipitations. La mémoire du sol est en revanche clairement visible en fin de sécheresse, avec des déficits d'humidité du sol encore élevés en septembre 1976. On peut enfin remarquer la corrélation spatiale très importante entre sécheresses agricole et hydrologique durant cet épisode, situation non généralisable aux autres événements de la période étudiée.

\section{III.3 IDENTIFICATION LOCALE ET NATIONALE DES ÉVÉNEMENTS}

Le choix d'un seuil d'état de sécheresse permet d'identifier les événements indépendants à l'échelle locale et de déterminer leurs caractéristiques, comme indiqué sur la Figure 5. Cet exemple pour le SSWI3 d'une maille située près de Toulouse montre 3 événements franchissant le seuil de $5 \%$ sur les 10 dernières années et la manière dont sont définies les différentes caractéristiques événementielles : début de l'événement, durée, magnitude (valeur absolue de la somme des valeurs de l'indice durant l'événement, en mois) et sévérité (valeur absolue du minimum atteint). L'identification des événements de sécheresse peut alors être effectuée sur chacun des points de calcul, afin de mettre en lumière les spécificités régionales des caractéristiques des événements de sècheresse.

\section{III.4 COMPARAISON DES ÉVÉNEMENTS AU NIVEAU NATIONAL}

Une sécheresse se développe à la fois dans l'espace et dans le temps, et de tels événements sur les 50 dernières années à l'échelle de la France (Corse exclue) ont été identifiés à l'aide d'algorithmes de contiguité spatiale et de continuité temporelle inspirés de travaux réalisés sur les sécheresses aux États-Unis [12].

Chacun des événements peut alors être caractérisé à l'aide des propriétés définies plus haut auxquelles est rajoutée l'évolution de la surface affectée. On peut ainsi calculer pour chaque événement une durée moyenne, une superficie moyenne, ainsi qu'une magnitude totale, définie comme la valeur absolue de la somme des indices dans l'espace-temps de la sécheresse.

La Figure 6 compare ainsi les relations superficie-duréemagnitude pour chacun des événements identifiés à l'aide du SPI3 et du SSWI3. La comparaison de trois événements marquants récents montre la diversité de leurs caractéristiques : la sécheresse de 1976 est ainsi la plus longue en termes météorologiques, mais dans la moyenne pour l'aspect agricole. Les sécheresses agricoles de 2003 et surtout 19891990 sont quant à elles exceptionnelles de par leur durée et leur magnitude.

\section{LA PRÉVISION NUMÉRIQUE DU TEMPS AUX ÉCHÉANCES ETENDUES}

Le besoin d'anticipation des sècheresses et des étiages nécessite aussi de valoriser les différentes techniques de prévision numérique du temps aux échéances étendues. Ces techniques et les possibilités offertes varient fortement selon les échéances. 

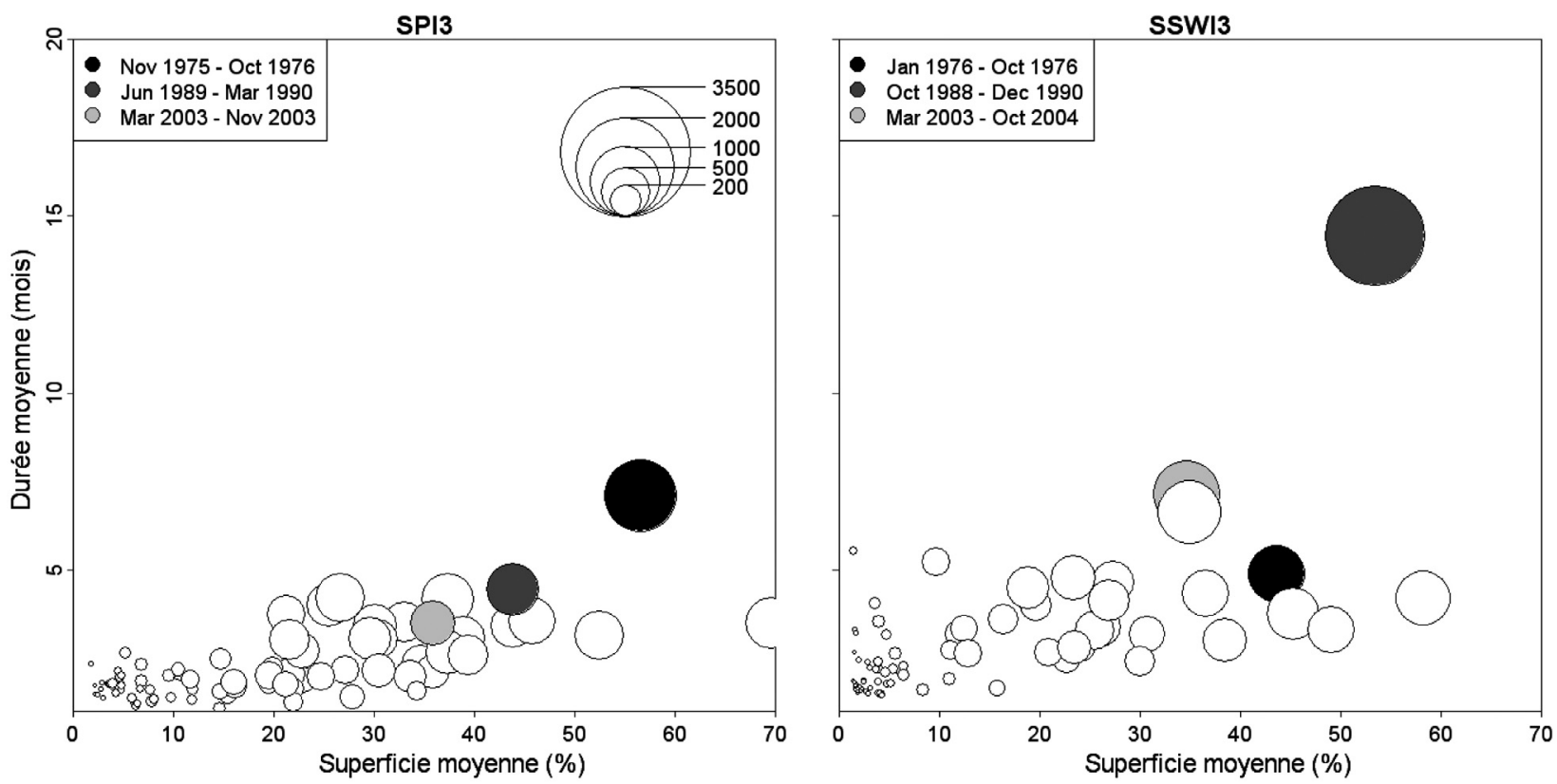

Figure 6 : Relations entre superficie moyenne (\% de la France), durée moyenne (mois) et magnitude totale (mois par pourcentage de la France) pour les événements de sécheresse indépendants identifiés avec le SPI3 et le SSWI3 et un seuil de probabilité de $20 \%$. L'aire de chaque disque est proportionnelle à la magnitude totale, dont l'échelle est donnée dans le graphe de gauche. Trois événements importants sont identifiés par différents niveaux de gris.

\section{IV.1 LA MOYENNE ÉCHÉANCE}

Au delà de 3 à 4 jours, les méthodes de prévision numériques du temps purement déterministes atteignent leurs limites mais conservent un caractère opérationnel grâce à l'utilisation de méthodes adaptées, dites ensemblistes, basées sur la prise en compte des incertitudes dans l'état initial de l'atmosphère. Ainsi, l'analyse du modèle est perturbée 50 fois sur des zones sensibles (peu d'observations et présence de forts gradients) et tient compte des incertitudes dans la représentation des processus physiques. On peut examiner en priorité le comportement de la moyenne des 51 prévisions (50 prévisions perturbées et la prévision déterministe) qui s'avère statistiquement la plus proche de la réalité et étudier ensuite les champs de probabilités associés à des franchissements de seuils de température ou de précipitations. Des visualisations de ces probabilités ont été développées pour faciliter l'expertise des prévisionnistes. Ainsi les produits de type " panache », comme le montre la figure 7, permettent d'apprécier la dispersion des prévisions, corrélée à la prévisibilité de la situation.

Des progrès importants ont été obtenus ces dernières années dans la performance de ces modèles grâce à la forte augmentation de la puissance de calcul disponible et à l'enrichissement continu des modèles numériques (physique amé-

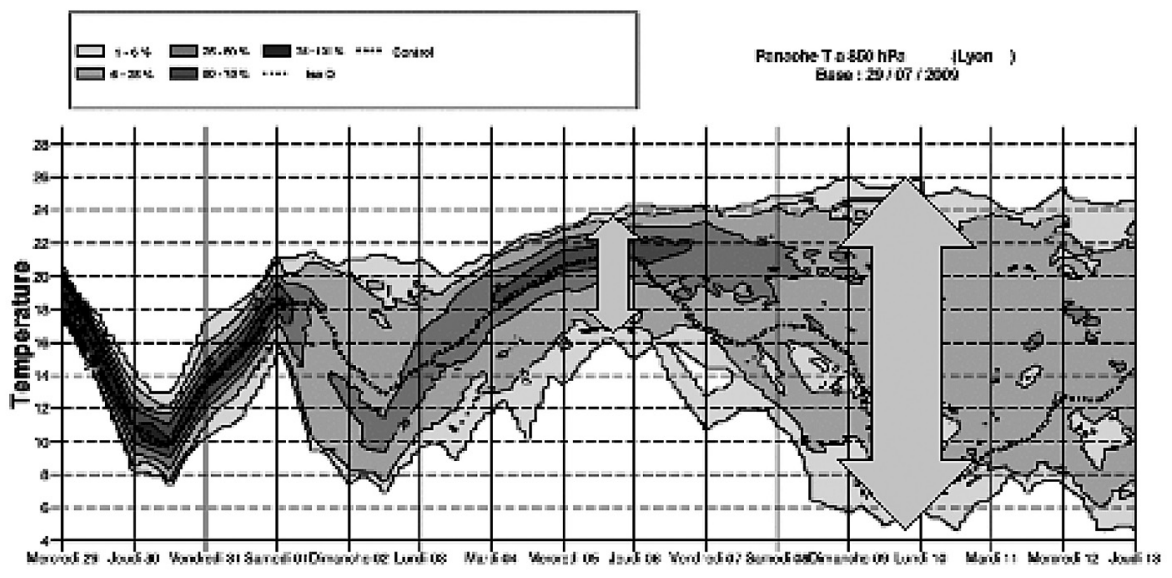

Figure 7 : Panache de température prévue à Lyon au niveau 850 hPa du mercredi 29 juillet jusqu'au jeudi 13 août 2009 : les flèches verticales visualisent la variabilité temporelle de la dispersion du panache. 
liorée, résolutions augmentées). À ce jour, la qualité de la prévision d'ensemble répond aux exigences de la prévision opérationnelle jusqu'aux échéances de 7 à 10 jours et peut permettre d'identifier la poursuite de conditions de sècheresse à l'échelle régionale ou le risque d'occurrence d'un événement de type canicule.

\section{IV.2 À L'ÉCHÉANCE MENSUELLE}

Pour aborder l'échéance mensuelle, il convient de prendre en compte le couplage de l'océan avec l'atmosphère. Une production opérationnelle est assurée depuis plusieurs années à un rythme hebdomadaire par le CEPMMT et fait l'objet d'une exploitation par Météo-France qui établit des prévisions pour quelques utilisateurs, dont par exemple EDF pour l'anticipation de la demande en énergie. Les principaux produits associés à cette chaîne de prévision sont des anomalies de températures, de champs de pression, de précipitations et leurs probabilités (semaine par semaine) sur le mois à venir. Ces produits peuvent permettent notamment d'identifier un risque régional de canicule sur la France à partir d'un signal de forte anomalie de température. Les vérifications effectuées a posteriori montrent que la prévisibilité des températures est plus élevée que celle des précipitations. Dans des situations de faible prévisibilité (peu de signal significatif), il peut être utile d'appliquer des méthodes basées sur la détermination du (ou des) régime(s) de temps le(s) plus probable(s) au cours des jours et surtout des semaines qui viennent. Il a été montré que, été comme hiver, 4 régimes de temps pilotent $90 \%$ des situations météorologiques sur la façade atlantique de l'Europe en lien avec la variabilité climatique tropicale à l'échelle intra saisonnière. Pour chaque régime de temps, il a pu être établi les caractéristiques moyennes des normales de précipitation et température ainsi que les probabilités d'occurrence de phénomènes extrêmes [13]. Ces informations sont utilisées en opérationnel pour l'analyse des scénarios prévisionnels.

\section{- IV.3 À L'ÉCHÉANCE SAISONNIÈRE}

La prévision saisonnière se situe résolument dans le domaine de la prévision du climat. Elle nécessite la modélisation de l'ensemble du système planétaire et la représentation des couplages entre les différents compartiments : océans, atmosphère, sols et végétation. Elle fait l'objet de nombreux développements et applications opérationnelles à travers le monde couvrant des horizons temporels de 3 à 6 mois, voire au-delà. La présentation des résultats fait référence essentiellement à des probabilités d'anomalie de températures et de précipitations à grande échelle, exploitant les statistiques du système de modélisation, acquises sur une période de référence longue, et situant ensuite chaque simulation par rapport à celles-ci. Des travaux sont menés pour améliorer ces systèmes de modélisation et enrichir les diagnostics qui permettent d'extraire l'information d'évolution climatique utile, par exemple en exploitant la notion de régimes de temps. La figure 8 illustre la prévisibilité particulière du régime $\mathrm{NAO}^{-}$sur l'Atlantique Nord au début d'année 2010.

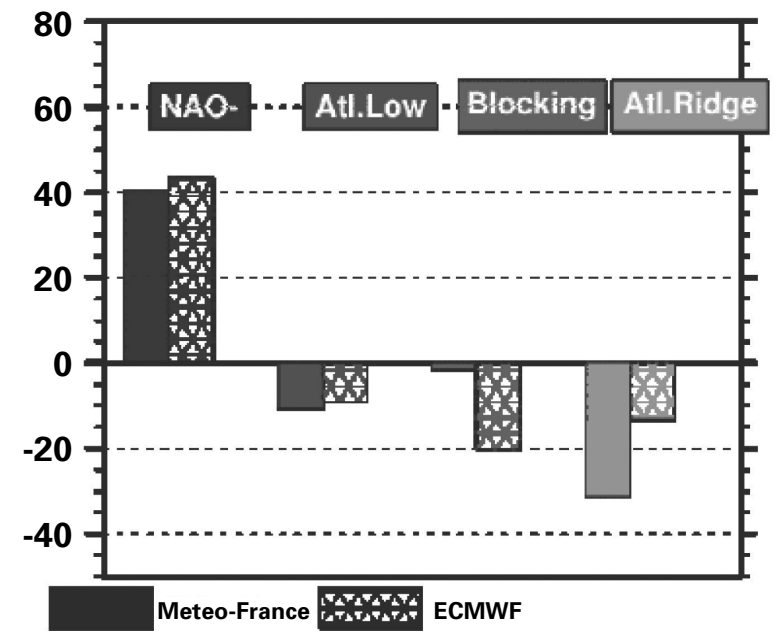

Figure 8 : Prévision de l'anomalie des occurrences des régimes de temps sur l'Atlantique Nord pour la période mars/avril/mai 2010 par les modèles de Météo-France et du CEPMMT (ECMWF)

Si la prévision saisonnière a prouvé son potentiel par des applications très concrètes dans la bande tropicale et certaines régions du globe en téléconnexion directe avec les bassins océaniques tropicaux, son maniement demeure plus délicat en d'autres endroits. Ainsi, la tâche reste difficile sur l'Europe de l'ouest qui reste une des régions du monde où la prévisibilité est la plus faible.

Météo-France, à l'instar des autres principaux services météorologiques, produit chaque mois un bulletin de prévision pour le trimestre suivant, disponible sur son site Internet, concernant la Métropole et les territoires d'Outre-mer. Cette production résulte d'une analyse exploitant les simulations des principaux centres producteurs (Météo-France, CEPMMT, NOAA, Met Office ...) et concrétise l'investissement consenti de longue date par les équipes de recherche sur le climat

\section{D PERSPECTIVES EN MATIÈRE DE PRÉVISION SAISONNIÈRE HYDROLOGIQUE}

Le couplage des modèles de prévision d'ensemble météorologique et des modèles hydrologiques fait également l'objet d'un effort de recherche important car il permet de prendre en compte les incertitudes liées à la prévision des précipitations dans les modèles hydrologiques, comme cela a été montré en matière de prévision des crues [14]. Un couplage de la chaîne SIM à la prévision d'ensemble EPS du CEPMMT a ainsi été développé en 2007 à Météo-France et permet de disposer pour la prévision des crues d'une chaine de prévision d'ensemble des débits à 10 jours d'échéance sur l'ensemble du territoire [15]. Son application à la prévision des bas débits nécessite cependant une réflexion approfondie 

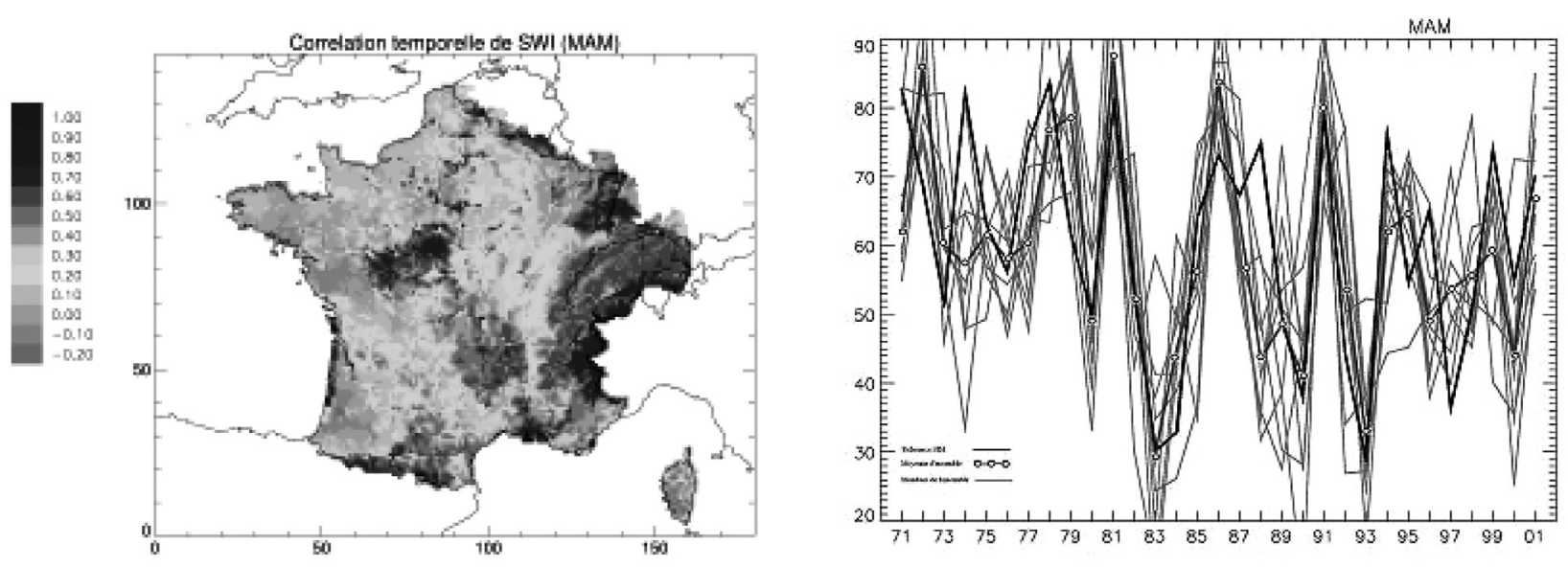

Figure 9 : Représentation spatiale de la corrélation temporelle (période 1971-2001) entre la moyenne trimestrielle de l'indice d'humidité des sols prévue (SWI) et celle de la référence SIM (à gauche) et comparaison des débits $\left(\mathrm{m}^{3} / \mathrm{s}\right)$ observés (foncé) et prévus par la moyenne d'ensemble (clair) sur le bassin de l'Ariège à Foix.pour le trimestre Mars-Avril-Mai sur la période 1971-2001.

sur les améliorations à apporter au système ne prenant pas en compte à ce jour les effets anthropiques.

Mais en matière de prévision hydrologique de la ressource en eau, la priorité est portée aujourd'hui à Météo-France sur l'échéance saisonnière sur la base d'une meilleure prévisibilité des paramètres hydrologiques de surface par rapport aux paramètres atmosphériques, et des travaux sur la prévision saisonnière des débits menés ces dernières années aux ÉtatsUnis, ainsi qu'en France [16]. Une étude a été conduite récemment sur la faisabilité et les potentialités du couplage entre les prévisions saisonnières et le modèle hydrologique SIM sur la France [17].

Le chaîne de prévision mise en place consiste à remplacer le module SAFRAN, habituellement utilisé en mode analyse pour les paramètres de surface, par les données de prévision saisonnière du projet européen DEMETER ${ }^{1}$ sur les années 1971-2001. La période du printemps a été choisie, notamment en regard de l'influence de la fonte nivale et des enjeux de l'anticipation des débits d'étiage. Le principe de cette chaîne est assez simple ; les données de températures et de précipitations sont issues des prévisions saisonnières alors que les autres paramètres atmosphériques, dans un premier temps, sont issus de la climatologie Safran. Les conditions initiales de surface et des débits au $1^{\text {er }}$ février sont fournis par la chaîne SIM analyse. Le modèle tourne alors sur une période de 120 jours à partir du premier février de façon à simuler les conditions du printemps (mars à mai). Cependant, les données DEMETER, issues de modèles de climat globaux, ne sont pas utilisables directement par le modèle Isba et nécessitent de passer d'une résolution de $2,5^{\circ}$ (environ $200 \mathrm{~km}$ ) à une résolution de $8 \mathrm{~km}$, compatible avec Isba. La méthode de descente d'échelle choisie est une adaptation de celle utilisée pour la prévision d'ensemble à moyen terme

1. DEMETER : Development of a European Multimodel model Ensemble system for seasonal to inTER annual prediction, cofinancé en 2004 par l’Union Européenne des débits utilisant les données du CEPMMT [18]. La qualité des forçages atmosphériques n'est pas dégradée par la descente d'échelle. On ne note pas de biais particulier sur les températures, les précipitations totales sont correctement estimées en quantité (sans présager de la qualité de la représentation de la variabilité interannuelle) mais avec une surestimation de la pluie et une sous-estimation de la neige.

Les résultats préliminaires sur l'indice d'humidité des sols sont très intéressants. Sur notre territoire on obtient des corrélations positives souvent supérieures à 0,3 , comme le montre la figure 9 avec des zones de prévisibilité maximum en montagne (influence de la neige) mais aussi sur certaines zones de plaine en fonction de la texture des sols. Une étude des potentialités en termes de prévision des débits a été menée sur 4 stations aux régimes hydrologiques différents : la Durance à Embrun, l'Ariège à Foix, la Seine à Paris et la Garonne à Tonneins. Comme pour l'humidité des sols les résultats sont encourageant, même si l'on peut probablement séparer les bassins avec des régimes de fonte nivale des autres ; les premiers offrant une plus grande prévisibilité liée notamment à l'effet du manteau neigeux. Les débits modélisés de 1'Ariège, présentés en figure 9, sont du même ordre de grandeur que les réanalyses SIM.

Outre la représentation correcte de la variabilité inter annuelle (corrélation stable voisine de 0,65 ), on peut aussi noter que la dispersion des membres individuels de l'ensemble couvre bien la gamme des valeurs de la référence SIM. Si l'on s'intéresse à des catégories (un peu) plus extrêmes $(\sim 20 \%)$ que les terciles, on retrouve une prévisibilité correcte pour ces catégories avec une bonne stabilité des modèles d'analyse discriminante débits prévus / débits observés. Enfin, il faut signaler que le signal intra-saisonnier (moyennes mensuelles), non encore étudié à ce niveau, semble aussi offrir des possibilités intéressantes tant au niveau de l'humidité des sols que du débit des rivières. Les travaux de recherche vont se poursuivre pour aller vers une version opérationnelle de cette chaîne qui pourrait fournir tous les 
mois des ensembles d'au moins 41 membres (actuellement pour l'atmosphère) sur une échéance de 7 mois.

\section{VI — CONCLUSIONS}

Plusieurs applications opérationnelles en lien étroit avec la recherche sont en cours de développement à Météo-France pour améliorer les capacités de caractérisation et prévision des situations de sècheresse et d'étiage. Ces applications s'appuient principalement sur la valorisation de la chaîne SIM, bien adaptée au suivi de la ressource en eau et à la construction d'indicateurs de sècheresse pour les différentes composantes du cycle de l'eau. Des expérimentations coordonnées par la DGALN et Météo-France sont en cours pour faciliter l'utilisation opérationnelle de ces informations dans le cadre du suivi hydrologique opérationnel des services de l'Etat (DREAL) et les discussions au sein des différents comités de sècheresse.

La prévision de l'évolution de la situation aux échéances étendues (semaine, mois, saison) rentre progressivement dans une phase pré-opérationnelle avec des outils qui permettent d'explorer les différentes sources de prévisibilité. Cette démarche présente un potentiel supérieur aux approches probabilistes basées sur les seules informations climatologiques. Notamment, le couplage de SIM avec les systèmes de prévision saisonnière vise à développer les bases d'un futur système opérationnel de prévision saisonnière hydrologique. L'ensemble de ces actions s'inscrit dans une dynamique plus large encore de compréhension de l'évolution du climat, de sa variabilité naturelle et de ses changements anthropiques dont un des éléments clés du système, le cycle hydrologique, est aussi un des sujets majeurs des enjeux du changement climatique et de ses impacts.

\section{VII — RÉFÉRENCES}

[1] Blanchard M., Franchisteguy L., Habets F., Martin E., Noilhan J. (2007) - Typologie des sècheresses sur la France et outils de suivi de la ressource en eau. Revue Française de géotechnique. 120-121 11-21

[2] Vidal J.-P., Moisselin J.-M. (2008) - Impact du changement climatique sur les sécheresses en France. Actes du XXI colloque de l'Association Internationale de Climatologie. Climat et risques climatiques en Méditerranée (Vinet, F., ed.). 655-660

[3] Vidal J.-P., Soubeyroux J.-M. (2008) - Impact du changement climatique en France sur la sécheresse et l'eau du sol. SEC 2008 - Symposium International - Sécheresse et Constructions (Magnan, J.-P., Cojean, R., Cui, Y.-J. \& Mestat, P., eds.). 1 25-31

[4] Habets F., Boone A., Champeaux J.-L., Etchevers P., Franchisteguy L., Leblois E., Ledoux E., Le Moigne P., Martin E., Morel S., Noilhan J., Quintana Segui P., Rousset-Regimbeau F., VienNot P. (2008) — The SAFRAN-
ISBA-MODCOU hydrometeorological model applied over France. Journal of Geophysical Research. 113 D06113

[5] Quintana-Segui P., Le Moigne P., Durand Y., Martin E., Habets F., Baillon M., Canellas C., Franchisteguy L., Morel S. (2008) - Analysis of near-surface atmospheric variables : Validation of the SAFRAN analysis over France. Journal of Applied Meteorology and Climatology. 47 92-107

[6] Vidal J.-P., Martin E., Franchisteguy L., Baillon M., SoubeyrouX J.-M. (2010) - A 50 year high-resolution atmospheric reanalysis over France with the Safran System. International Journal of Climatology. 30 1627-1644

[7] Soubeyroux J.-M., Martin E, Franchisteguy L., Habets F., Noilhan J., Baillon M., Regimbeau F., Vidal J.-P., Le Moigne P., Morel S. (2008) - SIM, un outil pour le suivi hydrométéorologique opérationnel et les études. $L a$ Météorologie. 63 40-45

[8] Viel C., Gouin P., Louis O., Alot C. (2008) - Safran-IsbaModcou (SIM) et la prévision des crues. La Météorologie. 63 46-51

[9] Wilhite D. A., Glantz M. H. (1985) — Understanding the drought phenomenon : The role of definitions. Water International. 10 111-120

[10] Омм (2009) - Communiqué de presse de l'Organisation Météorologique Mondiale $n^{\circ} 872$ du 15/12/2009.

[11] Vidal J.-P., Martin E., Franchisteguy L., Habets F., Soubeyroux J.-M., Blanchard M., Baillon M. (2010) Multilevel and multiscale drought reanalysis over France with the Safran-Isba-Modcou hydrometeorological suite. Hydrology and Earth System Sciences. 14 459-478

[12] Andreadis K. M., Clark E. A., Wood A. W., Hamlet A. F., LetTENMAiER D. P. (2005) - Twentieth-century drought in the conterminous United States. Journal of Hydrometeorology. 6 985-1001

[13] CAssou C. (2008) - Intraseasonal interaction between the Madden-Julian Oscillation and the North Atlantic Oscillation. Nature. 455 523-527

[14] Ramos M.H, Thielen J., Pappenberger F. (2008) — Utilisation de la prévision météorologique d'ensemble pour la prévision hydrologique opérationnelle et l'alerte aux crues. Actes du colloque SHF Prévisions hydrométéorologiques.

[15] Martin E., Thirel G., Regimbeau F., Noilhan J., Franchisteguy L., Soubeyroux J.-M. (2009) — Vers une prévision d'ensemble des débits à l'échelle des grands bassins français. DOI : 10.1051/1hb/2009060. La Houille Blanche. 5 88-94

[16] Sauquet E., Lerat J., Prudhomme C. (2007) - La prévision hydrométéorologique à 3-6 mois - État des connaissances et applications. DOI : 10.1051/lhb:2008075. La Houille Blanche. $677-84$

[17] Ceron J.-P., Tanguy G., Franchisteguy L., Martin E., Regimbeau F., Vidal J.-P. (2010) - Hydrological seasonal forecast over France : feasibility and prospects. Atmospheric Science Letters. 11 78-82

[18] Rousset-Regimbeau F. (2007) - Modélisation des bilans de surface et des débits sur la France, application à la prévision d'ensemble des débits. Thèse de doctorat, Université Paul Sabatier Toulouse. $215 \mathrm{p}$ 